\title{
Atrial Arrhythmia Related Outcomes in Critically Ill COVID-19 Patients
}

\author{
Chad Colon ${ }^{1}$, James Barrios ${ }^{1}$, Joe Chiles ${ }^{1}$, Todd Brown ${ }^{1}$, Steven Pogwizd ${ }^{1}$, Samuel \\ McElwee $^{1}$, Sheetal Gandotra ${ }^{1}$, Derek Russell ${ }^{1}$, Hugh McElderry ${ }^{1}$, and William Maddox ${ }^{1}$ \\ ${ }^{1}$ The University of Alabama at Birmingham
}

October 5, 2020

\begin{abstract}
Background: Coronavirus Disease 2019 (COVID-19) is associated with many clinical manifestations including respiratory failure and cardiovascular compromise. Objectives: We examine outcomes in critically ill individuals with COVID-19 who develop atrial tachyarrhythmias (ATA). Methods: We collected data from electrocardiograms and the electronic medical record of COVID19 positive (COVID+) and negative (COVID-) individuals admitted to our medical intensive care unit between February 29 and June 28, 2020. We compared clinical and demographic characteristics, new onset ATA, hemodynamic compromise (HC) following ATA, and in-hospital mortality in those who were COVID+ vs. COVID-. HC was defined as having a new or increased vasopressor requirement or the need for direct current cardioversion for hemodynamic instability within 1 hour of ATA onset. Results: Of 300 individuals included, 200 were COVID+ and 100 were COVID-. Mean age was $60 \pm 16$ years, 180 $(60 \%)$ were males, and 170 (57\%) were African American. New onset ATA occurred in 16\% of COVID+ and 19\% of COVIDindividuals $(\mathrm{p}=0.51)$. When compared to COVID- participants without ATA, COVID+ individuals with new onset ATA had higher mortality after multivariable adjustment (OR 5.0, 95\% CI 1.9-13.5). New onset ATA was followed by HC in 18 COVID+ but no COVID- participants $(\mathrm{P}=0.0001)$. COVID+ individuals with $\mathrm{HC}$ after ATA were requiring high levels of ventilatory support at the time of ATA onset. Conclusions: ATA may be an important mediator of HC in critically ill individuals with COVID-19, especially for those mechanically ventilated. Recognition of this could assist with clinical care and prognostication for individuals with COVID-19.
\end{abstract}

\section{Key Words:}

Atrial Arrhythmias, COVID19, Atrial Fibrillation, Atrial Flutter, Atrial Tachycardia

\section{Abbreviations}

ARDS - Acute respiratory distress syndrome

ATA - Atrial Tachyarrhythmia

COVID-19 - Coronavirus disease 2019

COVID $^{+}$- Coronavirus disease 2019 positive

COVID $^{-}$- Coronavirus disease 2019 negative

ECG - Electrocardiogram

$\mathrm{HC}$ - Hemodynamic compromise

HS Troponin - High sensitivity troponin

NE Eq - Norepinephrine equivalents 
PCR - Polymerase chain reaction

PEEP - Positive end expiratory pressure

UAB - University of Alabama at Birmingham

Introduction

Coronavirus Disease 2019 (COVID-19), the disease caused by the Severe Acute Respiratory Syndrome Coronavirus 2, can present with a wide range of clinical manifestations but most commonly with severe respiratory failure. ${ }^{1}$ A significant incidence of cardiovascular morbidity and mortality related to COVID-19 infection is now also recognized. ${ }^{2-5}$ We previously published an analysis showing a high incidence of atrial tachyarrhythmia (ATA) in critically ill COVID-19 individuals. ${ }^{6}$ This is similar to the incidence published in prior cohorts of critically ill patients without COVID-19. ${ }^{7}$ The development of new onset ATA has been associated with increased incidence of heart failure, stroke and death in critically ill patients. ${ }^{8-11}$ In this study, we report an analysis of consecutive critically ill participants that required intensive care unit (ICU) admission at a single center with a high degree of suspicion for COVID-19 infection. We describe the incidence of new-onset ATA and the association of ATA with short-term hemodynamic sequelae.

Methods

\section{Study Population}

We initially included 215 consecutive individuals age $>18$ years who were admitted to the medical intensive care unit (ICU) at the University of Alabama at Birmingham (UAB) Hospital that tested positive for COVID19 (COVID $^{+}$) between February 29 and June 28, 2020. In addition, we included 110 patients who were admitted with a suspicion of COVID-19 infection between March 13 and April 25, 2020 but who subsequently tested negative (COVID ${ }^{-}$) by nasopharyngeal swab polymerase chain reaction (PCR) assay. After excluding 15 patients who were $\mathrm{COVID}^{+}$and 10 individuals who were $\mathrm{COVID}^{-}$who had a diagnosis of permanent atrial fibrillation on admission, $200 \mathrm{COVID}^{+}$and $100 \mathrm{COVID}^{-}$patients were analyzed. Participants in each group were subsequently subclassified by the presence or absence of new onset ATA (Figure 1). The project was approved by the UAB Institutional Review Board with waiver of informed consent.

\section{Clinical data}

Participant demographic information, past medical history, comorbidities, inflammatory markers, high sensitivity (HS) troponin levels, outpatient medications, and inpatient therapies were collected from the electronic medical record. In addition, data on the need for mechanical ventilation, duration of mechanical ventilation, intensive care unit and hospital lengths of stay, and in-hospital mortality was obtained. To standardize vasopressor dosing across participants receiving different agents, the cumulative dose of norepinephrine equivalents (NE Eq) was calculated as has been described previously ${ }^{12,13}$ with a conversion factor for angiotensin II of $1 \mathrm{ng}$ per $0.1 \mathrm{mcg}$ of norepinephrine based upon the ATHOS-3 trial (Supplement Table 1). ${ }^{13}$ All 12 lead electrocardiograms (ECGs) recorded during admission were reviewed by a board certified cardiologist to determine the development of atrial arrhythmias. Participants with ECG documentation of a new-onset atrial fibrillation, atrial flutter, or atrial tachycardia were labeled as having an ATA.

\section{Outcomes}

We collected data on in-hospital mortality as well the development of hemodynamic compromise (HC) following ATA. To determine $\mathrm{HC}$ in those with new-onset ATA, the maximum NE Eq doses of vasopressor recorded the hour before the onset of ATA were compared with the maximum NE Eq dose of vasopressors during the hour after the onset of these arrhythmias. Participants were classified as having HC following ATA if their NE Eq vasopressor dose requirement increased or if direct current cardioversion was performed within one hour of acute arrhythmia onset.

\section{Statistical analyses}


Continuous variables are presented as mean \pm standard deviation and compared using independent samples t-tests. Categorical variables were expressed as frequencies and compared using chi-square tests. To examine mortality, we divided our study population into 4 groups: 1) COVID ${ }^{+}$with ATA, 2) COVID ${ }^{+}$without ATA, 3) COVID $^{-}$with ATA, and 4) COVID ${ }^{-}$without ATA. Individual logistic regression models were constructed using the COVID- without ATA group as the referent. Models were adjusted for age, sex, race, body mass index, and clinical characteristics that varied by COVID status (systolic heart failure, diastolic heart failure, atrial fibrillation, chronic kidney disease, diabetes, chronic obstructive pulmonary disease, cirrhosis, and tobacco use). All tests were two-tailed, and a $\mathrm{P}$ value $<.05$ (set a priori ) was considered statistically significant. All statistical analyses and graphics creations were performed using SPSS Statistics version 26 software (IBM Corp., Armonk, NY, USA) and GraphPad Prism v. 7.0 (GraphPad Software, San Diego, CA, USA).

\section{Results}

The baseline characteristics of those with and without COVID-19 are shown in Table 1. The cohort had many features that put them at high risk for COVID-19 complications with a mean age of $60 \pm 16$ years, 180 (60\%) were men, 170 (57\%) were African-American, 109 (36\%) were Caucasian, and there were high rates of underlying chronic metabolic, pulmonary, renal, and cardiovascular comorbidities that have been associated with poor outcome in other published COVID-19 cohorts. ${ }^{5,14-16}$ Compared to those who were COVID ${ }^{-}$, $\mathrm{COVID}^{+}$participants were more likely to have a history of diabetes $(47 \%$ vs. $34 \%, \mathrm{p}=0.04)$, but less likely to have a systolic heart failure ( $9 \%$ vs $27 \%, \mathrm{p}=0.001)$, diastolic dysfunction ( $17 \%$ vs $34 \%, \mathrm{p}=0.001)$, paroxysmal atrial fibrillation ( $5 \%$ vs. $14 \%, \mathrm{p}=0.004)$, chronic kidney disease ( $14 \%$ vs. $23 \%, \mathrm{p}=0.05)$, chronic obstructive pulmonary disease ( $12 \%$ vs. $30 \%, \mathrm{p}=0.0001)$, tobacco abuse $(26 \%$ vs $50 \%, \mathrm{p}=0.0001)$, and cirrhosis $(1 \%$ vs. $9 \%, \mathrm{p}=0.001)$. COVID ${ }^{+}$participants were less likely to be prescribed beta blockers in the outpatient setting $(28 \%$ vs. $47 \%, \mathrm{p}=0.001)$. COVID ${ }^{+}$participants also had higher D-dimer $(3542 \pm 4664 \mathrm{ng} / \mathrm{mL}$ vs. $1861 \pm$ $2290 \mathrm{ng} / \mathrm{mL}, \mathrm{p}=0.02)$ and CRP levels $(200 \pm 113 \mathrm{mg} / \mathrm{L}$ vs. $93 \pm 86 \mathrm{mg} / \mathrm{L}, \mathrm{p}=0.0001)$ but lower brain natriuretic peptide $(331 \pm 566 \mathrm{pg} / \mathrm{mL}$ vs. $732 \pm 812 \mathrm{pg} / \mathrm{mL}, \mathrm{p}=0.0001)$ and high sensitivity troponin (549 $\pm 1599 \mathrm{ng} / \mathrm{L}$ vs. $1906 \pm 7795 \mathrm{ng} / \mathrm{L}, \mathrm{p}=0.03)$ levels. Individuals with COVID-19 were more likely to require vasopressor support ( $71 \%$ vs. $38 \%, \mathrm{p}=0.0001)$ and mechanical ventilation $(75 \%$ vs. $40 \%, \mathrm{p}=0.0001)$ than those who were COVID $^{-}$. COVID ${ }^{+}$therapies included azithromycin in 150 participants, hydroxychloroquine in 7 participants, and remdesivir in 78 participants. An ATA was recorded by 12-lead ECG in 32 COVID $^{+}$

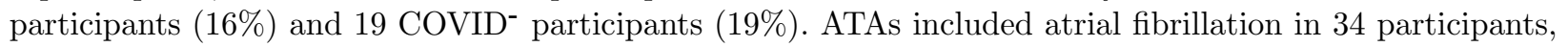
atrial flutter in 14 participants, and atrial tachycardia in 3 participants.

In-hospital mortality by COVID status in those with and without ATA is shown in Table 2. Individuals who were COVID ${ }^{+}$with new onset ATA had the highest in-hospital mortality $(50 \%)$ of any group $(\mathrm{p}=0.01)$. When compared to those who were $\mathrm{COVID}^{-}$without new onset ATA, individuals who were $\mathrm{COVID}^{+}$with new onset ATA had higher in-hospital mortality in both unadjusted (OR 4.4, 95\% CI 1.8 to 10.7) and multivariable adjusted (OR 5.0, 95\% CI 1.9 to 13.5) models. Individuals who were COVID $^{-}$with new onset ATA also had increased in-hospital mortality when compared to those who were COVID ${ }^{-}$without new onset ATA after multivariable adjustment (OR 2.3, 95\% CI 1.1 to 5.0). although the magnitude of this association was less than for those who were COVID $^{+}$with new onset ATA.

The demographic and clinical characteristics and inpatient therapies for individuals with ATAs are shown in Table 3. Those who were COVID $^{+}$and developed an ATA were more likely to require vasopressors $(91 \%$ vs $47 \%, \mathrm{p}=0.001$ ), had a longer duration on vasopressors ( $9 \pm 6$ vs $2 \pm 2$ days, $\mathrm{p}=0.0001$ ), were more likely to require mechanical ventilation ( $94 \%$ vs $42 \%, \mathrm{p}=0.0001$ ), had a longer duration of mechanical ventilation (18 \pm 11 vs $4 \pm 9$ days, $\mathrm{p}=0.0001$ ), had longer ICU lengths of stay (LOS) ( $23 \pm 8$ vs $12 \pm 11$ days, $\mathrm{p}=0.0001)$, and had longer hospital LOS ( $25 \pm 6$ vs $17 \pm 9$ days, $\mathrm{p}=0.0001$ ) compared to those who were COVID $^{-}$and developed an ATA.

$\mathrm{HC}$ occurred in 18 participants in the COVID ${ }^{+}$group and none in the COVID- group $(\mathrm{p}=0.0001)$. Among the $18 \mathrm{COVID}^{+}$individuals who experienced $\mathrm{HC}, 17$ experienced an increasing NE Eq requirement and 1 required immediate direct current cardioversion for hemodynamic instability at ATA onset. In the 17 participants that 
had an increase in vasopressor requirement, the average change in NE Eq was $0.18 \mu \mathrm{g} / \mathrm{kg} / \mathrm{min}$. A graphical representation of NE Eq dosage changes can be found in Figure 2. ATA was treated with amiodarone in 29 (57\%) participants, beta blockers in $38(75 \%)$, calcium channel blocker in $5(10 \%)$, and anticoagulation was felt to be safe in $31(61 \%)$ participants.

Characteristics of participants with new onset ATA by hemodynamic status are shown in Table 4. When compared to the 14 COVID $^{+}$hemodynamically stable participants following ATA onset, the 18 COVID $^{+}$participants who developed $\mathrm{HC}$ after ATA onset had similar comorbid conditions and baseline echocardiographic assessment with a lower mean arterial pressure $(74 \pm 16$ vs $89 \pm 10, \mathrm{p}=0.004)$, higher serum potassium $(4.5 \pm 0.4$ vs $4.2 \pm 0.5, \mathrm{p}=0.04)$, greater vasopressor use $(83 \%$ vs $21 \%, \mathrm{p}=0.0001)$, greater need for mechanical ventilation ( $100 \%$ vs $57 \%, \mathrm{P}=0.002)$, higher positive end expiratory pressure (PEEP) requirements ( $10 \pm 4$ vs $5 \pm 4, \mathrm{p}=0.005)$, and increased in-hospital mortality ( $67 \%$ vs. $29 \%, \mathrm{p}=0.03)$. In fact, of the 16 individuals with COVID-19 and a new onset ATA who subsequently died, 12 (75\%) had HC immediately after developing the ATA.

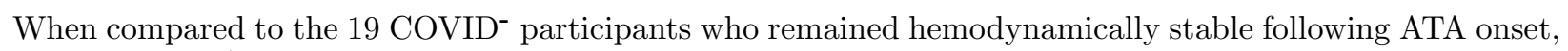
the 18 COVID $^{+}$participants who developed HC after ATA onset had a decreased prevalence of past diastolic dysfunction ( $11 \%$ vs. $47 \%, \mathrm{p}=0.02)$ and coronary artery disease $(11 \%$ vs. $42 \%, \mathrm{p}=0.03)$ but a higher serum potassium ( $4.5 \pm 0.4$ vs. $4.1 \pm 0.6, \mathrm{p}=0.02)$, greater need for vasopressor use $(83 \%$ vs. $11 \%, \mathrm{p}=0.0001)$, greater need for mechanical ventilation ( $100 \%$ vs. $16 \%, \mathrm{p}=0.0001)$, higher PEEP (10 \pm 4 vs. $1 \pm 3 \mathrm{~mm} \mathrm{Hg}$, $\mathrm{p}=0.0001)$, higher fraction of inspired oxygen requirements (57 \pm 17 vs. $29 \pm 6, \mathrm{P}=0.0001)$, and increased in-hospital mortality ( $67 \%$ vs. $29 \%, \mathrm{p}=0.01$ ).

\section{Discussion}

In this study, critically ill COVID ${ }^{+}$and COVID $^{-}$individuals with new onset ATA had increased in-hospital mortality when compared to those who were COVID- without ATA, although the magnitude of this association was greater for those who were $\mathrm{COVID}^{+}$. In addition, we observed a temporal relationship between new onset ATA and $\mathrm{HC}$ in individuals who were $\mathrm{COVID}^{+}$which might explain their increased in-hospital mortality. In fact, of the 16 individuals with COVID-19 and a new onset ATA who subsequently died, 12 (75\%) had HC immediately after developing the ATA.

ATA in critically ill individuals is thought to be driven by both individual factors such as myocardial dysfunction due to infection, drugs, and cytokine levels ${ }^{19}$ as well as by critical care interventions such as vasopressor use and mechanical ventilation. ${ }^{20-23}$ The occurrence of ATA during critical illness has been associated with poor outcomes, including increased hospital mortality ${ }^{9}$ increased duration of ICU admission, and 1-year adjusted survival. ${ }^{7}$ However, to our knowledge, the consequences of ATA in COVID-19 related critical illness have not been previously reported.

We found that the short-term effect of ATA on $\mathrm{COVID}^{+}$participants was distinct from that seen in those who were COVID- , with a marked temporal correlation between onset of ATA and HC seen uniquely among a group of $\mathrm{COVID}^{+}$individuals who were mechanically ventilated and requiring significant levels of ventilator support. COVID ${ }^{+}$participants who developed ATA with concurrent respiratory failure appeared much more vulnerable to HC just after ATA onset, suggesting an increased hemodynamic sensitivity of mechanically ventilated COVID ${ }^{+}$individuals to loss of sinus rhythm relative to COVID $^{-}$critically ill participants. Despite the known association of severe COVID-19 infection with cardiovascular comorbid diseases, COVID $^{+}$participants actually had a lower burden of chronic cardiac disease and valvular disease, and a higher ejection fraction compared to the COVID-group, arguing that structural heart disease is not the reason for their apparent hemodynamic sensitivity to the loss of sinus rhythm. Rather, our data suggest that the striking relationship of hemodynamic deterioration to new onset ATA in COVID ${ }^{+}$individuals may be related to cardiopulmonary interactions in severe acute respiratory distress syndrome (ARDS) and/or to the high degree of ventilator support that they require, including high PEEP support.

Previous studies have shown that increasing PEEP is associated with decreased cardiac output and mean blood pressure. ${ }^{24}$ We speculate that the loss of atrial contractility in individuals with COVID-19 ARDS 
may further decrease preload and cause hemodynamic decompensation. This is further supported by the high prevalence of mechanical ventilation and subsequent temporal decompensation observed at onset of ATA. Moreover, recent studies have highlighted the importance of right ventricular longitudinal strain in individuals with ARDS as a predictor of mortality highlighting the importance of right heart function and clinical outcomes. ${ }^{25,} 26$

These findings carry several important implications. This study suggests a potential causal relationship between ATA onset and hemodynamic instability in COVID ${ }^{+}$individuals. Importantly, the high mortality associated with ARDS appears to be driven more strongly by hemodynamic instability and degree of shock than by hypoxemia, ${ }^{27}$ therefore a complication so closely associated with marked hemodynamic deterioration may significantly influence outcomes. Indeed, participants with ATA associated HC did have worsened survival in our study. We hypothesize that vigilance to optimize factors that may increase the risk of ATA, such as electrolyte imbalances and volume overload, may be beneficial not only for heart rhythm, but also for blood pressure stability and downstream outcomes including survival. Although these findings may suggest that less hemodynamically impactful ventilatory strategies, such as a low PEEP strategy, could improve hemodynamic stability in COVID ${ }^{+}$individuals or ARDS individuals with ATA, this study does not directly address this question. It is conceivable that increased attention to a rhythm control strategy in COVID-19 individuals may have greater benefit than that seen in general critical illness, and prospective studies of this question may be justified. As our COVID- comparative cohort did not have a high incidence of ARDS, it is unclear if the observed hemodynamic changes related to ATA are unique to COVID infection and may represent a phenomenon seen in all individuals with severe ARDS. Studies have shown that prone positioning in individuals with ARDS improves ventilation and improves right ventricular ejection fraction, ${ }^{28}$ left ventricular preload ${ }^{28,29}$ and cardiac output. ${ }^{30,31}$ Thus, prone positioning may represent another potential approach to attenuate the hemodynamic effects of ATA in COVID-19, an effect which could conceivably contribute to the survival benefit shown with this agent in ARDS.

While the specific mechanism of myocardial injury in COVID infection remains to be defined, individuals susceptible to atrial arrhythmias and myocardial injury may be more likely to develop severe manifestations of viral infection. ${ }^{32}$ It remains to be seen whether early intervention of ATA in these individuals will mitigate the severe clinical course of the disease.

\section{Limitations}

There are several significant limitations of our study. First, this is a retrospective study from a single tertiary referral center which likely over represents individuals with severe manifestations of COVID-19 infection. Secondly, our study has a small sample size with a comparative group that does not perfectly match our $\mathrm{COVID}^{+}$population. However, our $\mathrm{COVID}^{+}$population had decreased incidence of comorbid conditions and structural heart abnormalities offering less potential for confounding than our standard intensive care population.

\section{Conclusions}

Individuals with COVID-19 who are admitted to the ICU have a high incidence of new onset ATA which is associated with HC and death. Special attention should be paid to new onset ATA in COVID ${ }^{+}$patients as it appears to be associated with worse outcomes.

\section{References:}

1. Bhatraju PK, Ghassemieh BJ, Nichols M, Kim R, Jerome KR, Nalla AK, Greninger AL, Pipavath S, Wurfel MM, Evans L, Kritek PA, West TE, Luks A, Gerbino A, Dale CR, Goldman JD, O'Mahony S and Mikacenic C. Covid-19 in Critically Ill Patients in the Seattle Region - Case Series. N Engl J Med . 2020.

2. Inciardi RM, Lupi L, Zaccone G, Italia L, Raffo M, Tomasoni D, Cani DS, Cerini M, Farina D, Gavazzi E, Maroldi R, Adamo M, Ammirati E, Sinagra G, Lombardi CM and Metra M. Cardiac Involvement in a Patient With Coronavirus Disease 2019 (COVID-19). JAMA Cardiol . 2020. 
3. Xu Z, Shi L, Wang Y, Zhang J, Huang L, Zhang C, Liu S, Zhao P, Liu H, Zhu L, Tai Y, Bai C, Gao T, Song J, Xia P, Dong J, Zhao J and Wang FS. Pathological findings of COVID-19 associated with acute respiratory distress syndrome. Lancet Respir Med . 2020.

4. Wang D, Hu B, Hu C, Zhu F, Liu X, Zhang J, Wang B, Xiang H, Cheng Z, Xiong Y, Zhao Y, Li Y, Wang $\mathrm{X}$ and Peng Z. Clinical Characteristics of 138 Hospitalized Patients With 2019 Novel Coronavirus-Infected Pneumonia in Wuhan, China. JAMA . 2020.

5. Shi S, Qin M, Shen B, Cai Y, Liu T, Yang F, Gong W, Liu X, Liang J, Zhao Q, Huang H, Yang B and Huang C. Association of Cardiac Injury With Mortality in Hospitalized Patients With COVID-19 in Wuhan, China.JAMA Cardiol . 2020.

6. Colon CM, JG B, JW C, SK M, DW R, WR M and GN K. Atrial Arrhythmias in COVID-19 Patients. JACC: Clinical Electrophysiology . 2020.

7. Klein Klouwenberg PM, Frencken JF, Kuipers S, Ong DS, Peelen LM, van Vught LA, Schultz MJ, van der Poll T, Bonten MJ, Cremer OL and * MC. Incidence, Predictors, and Outcomes of New-Onset Atrial Fibrillation in Critically Ill Patients with Sepsis. A Cohort Study. Am J Respir Crit Care Med . 2017;195:205211.

8. Santhanakrishnan R, Wang N, Larson MG, Magnani JW, McManus DD, Lubitz SA, Ellinor PT, Cheng S, Vasan RS, Lee DS, Wang TJ, Levy D, Benjamin EJ and Ho JE. Atrial Fibrillation Begets Heart Failure and Vice Versa: Temporal Associations and Differences in Preserved Versus Reduced Ejection Fraction. Circulation . 2016;133:484-92.

9. Moss TJ, Calland JF, Enfield KB, Gomez-Manjarres DC, Ruminski C, DiMarco JP, Lake DE and Moorman JR. New-Onset Atrial Fibrillation in the Critically Ill. Crit Care Med . 2017;45:790-797.

10. Shaver CM, Chen W, Janz DR, May AK, Darbar D, Bernard GR, Bastarache JA and Ware LB. Atrial Fibrillation Is an Independent Predictor of Mortality in Critically Ill Patients. Crit Care Med . 2015;43:210411.

11. Walkey AJ, Wiener RS, Ghobrial JM, Curtis LH and Benjamin EJ. Incident stroke and mortality associated with new-onset atrial fibrillation in patients hospitalized with severe sepsis. JAMA . 2011;306:224854.

12. Russell JA, Wellman H and Walley KR. Vasopressin versus norepinephrine in septic shock: a propensity score matched efficiency retrospective cohort study in the VASST coordinating center hospital.J Intensive Care . 2018;6:73.

13. Khanna A, English SW, Wang XS, Ham K, Tumlin J, Szerlip H, Busse LW, Altaweel L, Albertson TE, Mackey C, McCurdy MT, Boldt DW, Chock S, Young PJ, Krell K, Wunderink RG, Ostermann M, Murugan R, Gong MN, Panwar R, Hästbacka J, Favory R, Venkatesh B, Thompson BT, Bellomo R, Jensen J, Kroll S, Chawla LS, Tidmarsh GF, Deane AM and Investigators A-. Angiotensin II for the Treatment of Vasodilatory Shock. N Engl J Med . 2017;377:419-430.

14. Grasselli G, Zangrillo A, Zanella A, Antonelli M, Cabrini L, Castelli A, Cereda D, Coluccello A, Foti G, Fumagalli R, Iotti G, Latronico N, Lorini L, Merler S, Natalini G, Piatti A, Ranieri MV, Scandroglio AM, Storti E, Cecconi M, Pesenti A, Nailescu A, Corona A, Protti A, Albertin A, Forastieri Molinari A, Lombardo A, Pezzi A, Benini A, Malara A, Micucci A, Sala A, Alborghetti A, Antonini B, Capra C, Troiano C, Roscitano C, Radrizzani D, Chiumello D, Coppini D, Guzzon D, Costantini E, Malpetti E, Zoia E, Catena E, Agosteo E, Barbara E, Beretta E, Boselli E, Harizay F, Della Mura F, Lorini FL, Donato Sigurtà F, Marino F, Mojoli F, Rasulo F, Casella G, De Filippi G, Castelli G, Aldegheri G, Gallioli G, Lotti G, Albano G, Landoni G, Marino G, Vitale G, Battista Perego G, Evasi G, Citerio G, Merli G, Sforzini I, Bianciardi L, Carnevale L, Grazioli L, Guatteri L, Salvi L, Dei Poli M, Galletti M, Gemma M, Ranucci M, Riccio M, Borelli M, Zambon M, Subert M, Mazzoni MG, Raimondi M, Panigada M, Belliato M, Bronzini N, Petrucci N, Belgiorno N, Tagliabue P, Cortellazzi P, Gnesin P, Grosso P, Gritti P, Perazzo P, Severgnini P, Ruggeri P, 
Sebastiano P, Covello RD, Fernandez-Olmos R, Keim R, Rona R, Valsecchi R, Cattaneo S, Colombo, Cirri S, Bonazzi S, Greco S, Muttini S, Langer T, Alaimo V, Viola U and Network C-LI. Baseline Characteristics and Outcomes of 1591 Patients Infected With SARS-CoV-2 Admitted to ICUs of the Lombardy Region, Italy.JAMA . 2020.

15. Yu Y, Xu D, Fu S, Zhang J, Yang X, Xu L, Xu J, Wu Y, Huang C, Ouyang Y, Yang L, Fang M, Xiao H, Ma J, Zhu W, Hu S, Hu Q, Ding D, Hu M, Zhu G, Xu W, Guo J, Yuan H, Zhang B, Yu Z, Chen D, Yuan S and Shang Y. Patients with COVID-19 in 19 ICUs in Wuhan, China: a cross-sectional study. Crit Care . 2020;24:219.

16. Mehra MR, Desai SS, Kuy S, Henry TD and Patel AN. Cardiovascular Disease, Drug Therapy, and Mortality in Covid-19. N Engl J Med . 2020.

17. Zhou F, Yu T, Du R, Fan G, Liu Y, Liu Z, Xiang J, Wang Y, Song B, Gu X, Guan L, Wei Y, Li H, Wu $\mathrm{X}, \mathrm{Xu} \mathrm{J}$, Tu S, Zhang Y, Chen $\mathrm{H}$ and Cao B. Clinical course and risk factors for mortality of adult inpatients with COVID-19 in Wuhan, China: a retrospective cohort study. Lancet . 2020.

18. Liu K, Fang YY, Deng Y, Liu W, Wang MF, Ma JP, Xiao W, Wang YN, Zhong MH, Li CH, Li GC and Liu HG. Clinical characteristics of novel coronavirus cases in tertiary hospitals in Hubei Province. Chin Med $J$ (Engl) . 2020;133:1025-1031.

19. Bosch NA, Cimini J and Walkey AJ. Atrial Fibrillation in the ICU.Chest . 2018;154:1424-1434.

20. Seemann A, Boissier F, Razazi K, Carteaux G, de Prost N, Brun-Buisson C and Mekontso Dessap A. New-onset supraventricular arrhythmia during septic shock: prevalence, risk factors and prognosis.Ann Intensive Care . 2015;5:27.

21. Duarte PAD, Leichtweis GE, Andriolo L, Delevatti YA, Jorge AC, Fumagalli AC, Santos LC, Miura CK and Saito SK. Factors Associated with the Incidence and Severity of New-Onset Atrial Fibrillation in Adult Critically Ill Patients. Crit Care Res Pract . 2017;2017:8046240.

22. Rhodes A, Evans LE, Alhazzani W, Levy MM, Antonelli M, Ferrer R, Kumar A, Sevransky JE, Sprung CL, Nunnally ME, Rochwerg B, Rubenfeld GD, Angus DC, Annane D, Beale RJ, Bellinghan GJ, Bernard GR, Chiche JD, Coopersmith C, De Backer DP, French CJ, Fujishima S, Gerlach H, Hidalgo JL, Hollenberg SM, Jones AE, Karnad DR, Kleinpell RM, Koh Y, Lisboa TC, Machado FR, Marini JJ, Marshall JC, Mazuski JE, McIntyre LA, McLean AS, Mehta S, Moreno RP, Myburgh J, Navalesi P, Nishida O, Osborn TM, Perner A, Plunkett CM, Ranieri M, Schorr CA, Seckel MA, Seymour CW, Shieh L, Shukri KA, Simpson SQ, Singer M, Thompson BT, Townsend SR, Van der Poll T, Vincent JL, Wiersinga WJ, Zimmerman JL and Dellinger RP. Surviving Sepsis Campaign: International Guidelines for Management of Sepsis and Septic Shock: 2016. Intensive Care Med . 2017;43:304-377.

23. Vieillard-Baron A and Boyd J. Non-antiarrhythmic interventions in new onset and paroxysmal sepsisrelated atrial fibrillation.Intensive Care Med . 2018;44:94-97.

24. Jardin F, Farcot JC, Boisante L, Curien N, Margairaz A and Bourdarias JP. Influence of positive endexpiratory pressure on left ventricular performance. $N$ Engl J Med . 1981;304:387-92.

25. Li Y, Li H, Zhu S, Xie Y, Wang B, He L, Zhang D, Zhang Y, Yuan H, Wu C, Sun W, Zhang Y, Li M, Cui L, Cai Y, Wang J, Yang Y, Lv Q, Zhang L and Xie M. Prognostic Value of Right Ventricular Longitudinal Strain in Patients with COVID-19. JACC: Cardiovascular Imaging (2020). doi: https://doi.org/10.1016/j.jcmg.2020.04.014.

26. Argulian E, Sud K, B V, Bohra C, Garg V, Talebi S, Lerakis S and Narula J. Right Ventricular Dilation in Hospitalized Patients with COVID-19 Infection. JACC: Cardiovascular Imaging ( 2020). doi: https://doi.org/10.1016/j.jcmg.2020.05.010.

27. Vieillard-Baron A, Girou E, Valente E, Brun-Buisson C, Jardin F, Lemaire F and Brochard L. Predictors of mortality in acute respiratory distress syndrome. Focus On the role of right heart catheterization. $A m J$ 
Respir Crit Care Med . 2000;161:1597-601.

28. Vieillard-Baron A, Charron C, Caille V, Belliard G, Page B and Jardin F. Prone positioning unloads the right ventricle in severe ARDS. Chest . 2007;132:1440-6.

29. Jozwiak M, Teboul JL, Anguel N, Persichini R, Silva S, Chemla D, Richard C and Monnet X. Beneficial hemodynamic effects of prone positioning in patients with acute respiratory distress syndrome.Am J Respir Crit Care Med . 2013;188:1428-33.

30. Ruste M, Bitker L, Yonis H, Riad Z, Louf-Durier A, Lissonde F, Perinel-Ragey S, Guerin C and Richard JC. Hemodynamic effects of extended prone position sessions in ARDS. Ann Intensive Care . 2018;8:120.

31. Hering R, Wrigge H, Vorwerk R, Brensing KA, Schröder S, Zinserling J, Hoeft A, Spiegel TV and Putensen C. The effects of prone positioning on intraabdominal pressure and cardiovascular and renal function in patients with acute lung injury. Anesth Analg . 2001;92:1226-31.

32. Clerkin KJ, Fried JA, Raikhelkar J, Sayer G, Griffin JM, Masoumi A, Jain SS, Burkhoff D, Kumaraiah D, Rabbani L, Schwartz A and Uriel N. Coronavirus Disease 2019 (COVID-19) and Cardiovascular Disease.Circulation . 2020.

Table 1: Demographic and Clinical Characteristics of Critically Ill Participants Overall and by COVID-19 Status, February - June 2020

Table 2: New onset Atrial Tachyarrhythmia

Total $\mathrm{n}=300$

$\mathrm{COVID}^{+} \mathrm{n}=200$

COVID $^{-} \mathrm{n}=100$

$\mathrm{p}$ value*

Age (years)

$60 \pm 16$

$60 \pm 16$

$60 \pm 16$

0.69

BMI $\left(\mathrm{kg} / \mathrm{m}^{2}\right)$

$31 \pm 12$

$32 \pm 9$

$30 \pm 17$

0.25

Male

$180(60 \%)$

$120(60 \%)$

$60(60 \%)$

1.0

Caucasian 
$109(36 \%)$

$61(31 \%)$

$48(48 \%)$

0.005

African American

$170(57 \%)$

$121(61 \%)$

$49(49 \%)$

Other

$21(7 \%)$

$18(9 \%)$

$3(3 \%)$

Clinical Characteristics

Clinical Characteristics

Clinical Characteristics

Clinical Characteristics

Clinical Characteristics

Hypertension

$205(68 \%)$

$143(72 \%)$

$62(62 \%)$

0.10

Systolic HF

$44(15 \%)$

$17(9 \%)$

$27(27 \%)$

0.0001

Diastolic HF

$68(23 \%)$

$34(17 \%)$

$34(34 \%)$

0.001

Coronary Artery Disease

$59(20 \%)$ 
$33(17 \%)$

$26(26 \%)$

0.05

Atrial Fibrillation

$23(7 \%)$

$9(5 \%)$

$14(14 \%)$

0.004

Diabetes

$127(42 \%)$

$93(47 \%)$

$34(34 \%)$

0.04

Chronic Kidney Disease

$51(17 \%)$

$28(14 \%)$

$23(23 \%)$

0.05

Stroke

$36(12 \%)$

$19(10 \%)$

$17(17 \%)$

0.06

COPD

$54(18 \%)$

$24(12 \%)$

$30(30 \%)$

0.0001

Cirrhosis

$10(3 \%)$

$1(1 \%)$

$9(9 \%)$

0.0001

Obstructive Sleep Apnea 
$35(12 \%)$

$23(12 \%)$

$12(12 \%)$

0.90

Tobacco Abuse

$102(34 \%)$

$52(26 \%)$

$50(50 \%)$

0.0001

Outpatient Medications

Outpatient Medications

Outpatient Medications

Outpatient Medications

Outpatient Medications

ACE-I or ARB

$85(28 \%)$

$56(28 \%)$

$29(29 \%)$

0.88

Beta-Blocker

$103(34 \%)$

$56(28 \%)$

$47(47 \%)$

0.001

Aspirin

$86(28 \%)$

$53(27 \%)$

$33(33 \%)$

0.25

P2Y12 Inhibitor

$25(8 \%)$

$15(8 \%)$

$10(10 \%)$

0.47 
Peak Inpatient Laboratory Values Peak Inpatient Laboratory Values Peak Inpatient Laboratory Values Peak Inpatient Laboratory Values Peak Inpatient Laboratory Values

D-Dimer (ng/mL)

$3100 \pm 4232$

$3542 \pm 4664$

$1861 \pm 2290$

0.02

CRP (mg/L)

$176 \pm 116$

$200 \pm 113$

$93 \pm 86$

0.0001

$\mathrm{BNP}(\mathrm{pg} / \mathrm{mL})$

$474 \pm 690$

$331 \pm 566$

$732 \pm 812$

0.0001

hs Troponin (ng/L)

$968 \pm 4557$

$549 \pm 1599$

$1906 \pm 7795$

0.03

Inpatient Therapies

Inpatient Therapies

Inpatient Therapies

Inpatient Therapies

Inpatient Therapies

Vasopressor Use

$180(60 \%)$

$142(71 \%)$

$38(38 \%)$ 
0.0001

Mechanical Ventilation

$190(63 \%)$

$150(75 \%)$

$40(40 \%)$

0.0001

Outcomes

Outcomes

Outcomes

Outcomes

Outcomes

New ATA

$51(17 \%)$

$32(16 \%)$

$19(19 \%)$

0.51

Death

$86(29 \%)$

$66(33 \%)$

$20(20 \%)$

0.02

Values are mean $\pm S D$ or $n(\%)$.

${ }^{*} p$ value comparing $\mathrm{COVID}^{+}$vs. COVID ${ }^{-}$.

ACE-I, Angiotensin converting enzyme inhibitor; ARB, angiotensin receptor blocker; ATA, atrial tachyarrhythmia; BMI, body mass index; BNP, brain natriuretic peptide; COPD, chronic obstructive pulmonary disease; CRP, c-reactive protein; COVID, coronavirus disease; Diastolic HF, diastolic heart failure; hs Troponin, high sensitivity troponin; Systolic HF, systolic heart failure;

Table 2: In-Hospital Mortality by COVID Status in those with and without New Onset Atrial Tachyarrhythmia, February - June 2020

Table 2

Unadjusted

Unadjusted

Unadjusted

Unadjusted

Adjusted*

Adjusted* 
Adjusted*

Adjusted*

Adjusted*

Adjusted*

Adjusted*

95\% C.I.

95\% C.I.

95\% C.I.

95\% C.I.

95\% C.I.

95\% C.I.

95\% C.I.

Odds Ratio

Lower

Upper

Odds Ratio

Odds Ratio

Lower

Lower

Lower

Upper

Upper

Upper

COVID $^{+}$ATA

$16(50 \%)$

4.4

1.8

10.7

5.0

5.0

5.0

1.9

1.9

1.9 
13.5

COVID $^{+}$NO ATA

$50(30 \%)$

1.9

1.0

3.6

1.6

1.6

1.6

0.4

0.4

0.4

5.7

COVID' ATA

$5(26 \%)$

1.5

0.5

5.0

2.3

2.3

2.3

1.1

1.1

1.1

5.0

COVID ${ }^{-}$NO ATA

$15(19 \%)$

Referent

Referent

Referent

Referent

Referent

Referent

Referent 
Referent

Referent

Referent

Referent

*Adjusted for age, gender, race, body mass index, systolic heart failure, diastolic heart failure, atrial fibrillation, chronic kidney disease, diabetes, chronic obstructive pulmonary disease, cirrhosis, and tobacco use

ATA, atrial tachyarrhythmia; C.I., confidence interval; COVID, coronavirus disease

Table 3: Demographics and Clinical Characteristics of Critically Ill Participants with New Onset Atrial Tachyarrhythmias by COVID-19 Status, February - June 2020

Table 2

$\mathrm{COVID}^{+}$ATA $\mathrm{n}=32$

COVID $^{-}$ATA $n=19$

p Value

Age (years)

$65 \pm 13$

$64 \pm 15$

0.88

BMI $\left(\mathrm{kg} / \mathrm{m}^{2}\right)$

$29 \pm 8$

$27 \pm 7$

0.37

Male

$21(66 \%)$

$12(63 \%)$

0.82

White

$15(47 \%)$

$11(58 \%)$

0.45

Inpatient Therapies

Inpatient Therapies

Inpatient Therapies

Inpatient Therapies

Vasopressor Use 
$29(91 \%)$

$9(47 \%)$

0.00

Vasopressor Days

$9 \pm 6$

$2 \pm 2$

0.0001

Max NE Eq $(\mu \mathrm{g} / \mathrm{kg} / \mathrm{min})$

$0.45 \pm 0.51$

$0.28 \pm 0.43$

0.22

Mechanical Ventilation

$30(94 \%)$

$8(42 \%)$

0.0001

Days on MV

$18 \pm 11$

$4 \pm 9$

0.0001

Outcomes

Outcomes

Outcomes

Outcomes

ICU LOS (Days)

$23 \pm 8$

$12 \pm 11$

0.0001

Hospital LOS (Days)

$25 \pm 6$

$17 \pm 9$

0.0001

Death

$16(50 \%)$

$5(26 \%)$ 
Values are mean $\pm S D$ or $n(\%)$.

ATA, atrial tachyarrhythmia; BMI, body mass index; COVID, coronarvirus disease; Hospital LOS, hospital length of stay; ICU LOS, intensive care unit length of stay; Max, maximum; MV, mechanical ventilation; NE Eq, norepinephrine equivalents

Table 4: Demographic and Clinical Characteristics of Critically Ill Participants who were Hemodynamically Stable or Unstable Following Atrial Tachyarrhythmia Onset by COVID19 Status, February - June 2020

Table 2: New onset Atrial Tachyarrhythmia

$\mathrm{COVID}^{+} \mathrm{n}=32$

$\mathrm{COVID}^{+} \mathrm{n}=32$

COVID $^{-} \mathrm{n}=19$

$\mathrm{HC} n=18$

Stable $\mathrm{n}=14$

p Value*

Stable $n=19$

p Value ${ }^{+}$

Age (years)

$64+-15$

$62+-12$

0.63

$64+-15$

0.92

BMI $\left(\mathrm{kg} / \mathrm{m}^{2}\right)$

$30+-8$

$27+-6$

0.27

$27+-7$

0.24

Male

$11(61 \%)$

$10(71 \%)$

0.54

$12(63 \%)$

0.90

White 
$9(50 \%)$

$8(57 \%)$

0.69

$11(58 \%)$

0.63

Past Medical History

Past Medical History

Past Medical History

Past Medical History

Past Medical History

Past Medical History

Hypertension

$14(78 \%)$

$9(64 \%)$

0.40

$13(68 \%)$

0.19

Systolic HF

$2(11 \%)$

$3(21 \%)$

0.43

$5(26 \%)$

0.24

Diastolic HF

$2(11 \%)$

$4(29 \%)$

0.21

$9(47 \%)$

0.02

Coronary Artery Disease

$2(11 \%)$

$3(21 \%)$

0.43

$8(42 \%)$ 
0.03

Atrial Fibrillation

$1(6 \%)$

$3(21 \%)$

0.18

$5(26 \%)$

0.09

Diabetes

$10(56 \%)$

$4(29 \%)$

0.13

$6(32 \%)$

0.14

COPD

$3(17 \%)$

$2(14 \%)$

0.85

$7(37 \%)$

0.17

Echocardiography

Echocardiography

Echocardiography

Echocardiography

Echocardiography

Echocardiography

LVEF

$55+-14$

$58+-10$

0.62

$44+-17$

0.07

Diastolic Dysfunction

$4(22 \%)$

$3(30 \%)$ 
0.78

$11(92 \%)$

0.0001

LVIDd

$4.7+-0.8$

$4.9+-0.3$

0.41

$5.3+-1.4$

0.16

RV Dysfunction

$5(28 \%)$

$1(10 \%)$

0.21

$1(10 \%)$

0.21

TAPSE

$2.0+-0.4$

$2.2+-0.5$

0.38

$2.0+-0.4$

0.96

LA Dilation

$2(11 \%)$

$2(25 \%)$

0.65

$3(27 \%)$

0.54

Mitral Regurgitation

$3(17 \%)$

$4(29 \%)$

0.42

$5(26 \%)$

0.48

Tricuspid Regurgitation 
$5(28 \%)$

$6(43 \%)$

0.37

$7(37 \%)$

0.56

Aortic Regurgitation

$2(11 \%)$

0

0.20

$4(21 \%)$

0.41

Pericardial Effusion

$4(22 \%)$

$3(21 \%)$

0.96

$3(16 \%)$

0.62

Vital Signs and Laboratory Values at ATA Onset Vital Signs and Laboratory Values at ATA Onset Vital Signs and Laboratory Values at ATA Onset Vital Signs and Laboratory Values at ATA Onset Vital Signs and Laboratory Values at ATA Onset Vital Signs and Laboratory Values at ATA Onset Heart Rate Change

$50+-22$

$35+-24$

0.09

$39+-25$

0.18

MAP

$74+-16$

$89+-10$

0.004

$82+-15$ 
0.12

Sodium

$140+-6$

$137+-5$

0.34

$138+-8$

0.49

Potassium

$4.5+-0.4$

$4.2+-0.5$

0.04

$4.1+-0.6$

0.02

Magnesium

$2.0+-0.4$

$2.2+-0.4$

0.21

$2.0+-0.5$

0.99

Creatinine

$2.2+-1.4$

$1.8+-0.8$

0.46

$2.2+-2.4$

0.90

Inpatient Therapies at ATA Onset

Inpatient Therapies at ATA Onset

Inpatient Therapies at ATA Onset

Inpatient Therapies at ATA Onset

Inpatient Therapies at ATA Onset

Inpatient Therapies at ATA Onset

Mechanical Ventilation

$18(100 \%)$

$8(57 \%)$ 
0.002

$3(16 \%)$

0.0001

Prone Positioning

$2(11 \%)$

0

0.20

0

0.14

Vasopressors Use

$15(83 \%)$

$3(21 \%)$

0.0001

$2(11 \%)$

0.0001

CRRT

$9(50 \%)$

$4(29 \%)$

0.22

$4(21 \%)$

0.07

Ventilation Parameters at ATA Onset ${ }^{++}$

Ventilation Parameters at ATA Onset ${ }^{++}$

Ventilation Parameters at ATA Onset ${ }^{++}$

Ventilation Parameters at ATA Onset ${ }^{++}$

Ventilation Parameters at ATA Onset ${ }^{++}$

Ventilation Parameters at ATA Onset ${ }^{++}$

PEEP

$10+-4$

$5+-4$

0.005

$1+-3$

0.0001

Plateau Pressure 
$25+-7$

$23+-5$

0.58

$27+-4$

0.75

$\mathrm{FiO} 2(\%)$

$57+-17$

$46+-15$

0.06

$29+-6$

0.0001

Outcomes

Outcomes

Outcomes

Outcomes

Outcomes

Outcomes

Death

$12(67 \%)$

$4(29 \%)$

0.03

$5(26 \%)$

0.01

Values are mean +- SD or $\mathrm{n}(\%)$

${ }^{*} p$ Value comparing $\mathrm{COVID}^{+}$hemodynamic collapse group vs. $\mathrm{COVID}^{+}$stable group .

${ }^{+} p$ Value comparing $C \mathrm{OVID}^{+}$hemodynamic collapse group vs.COVID- stable group .

++ Individuals who were on room air at the time of analysis were given a PEEP value of 0 and an FiO2 value of $(0.21+$ Oxygen in $L$ per minute times 0.03). No plateau pressure was recorded for patients not ventilated.

ATA, atrial tachyarrhythmia; BMI, Body Mass Index; COPD, Chronic obstructive pulmonary disease; COVID, coronavirus disease; CRRT, continuous renal replacement therapy; FiO2, fraction of inspired oxygen; LA Dilation, left atrial dilation; LVEF, Left ventricular ejection fraction; LVIDd, Left ventricular internal diameter end diastole; MAP, Mean arterial pressure; PEEP, Positive end expiratory pressure; TAPSE, Tricuspid annular plane systolic excursion

Figure 1:

Enrollment Diagram 


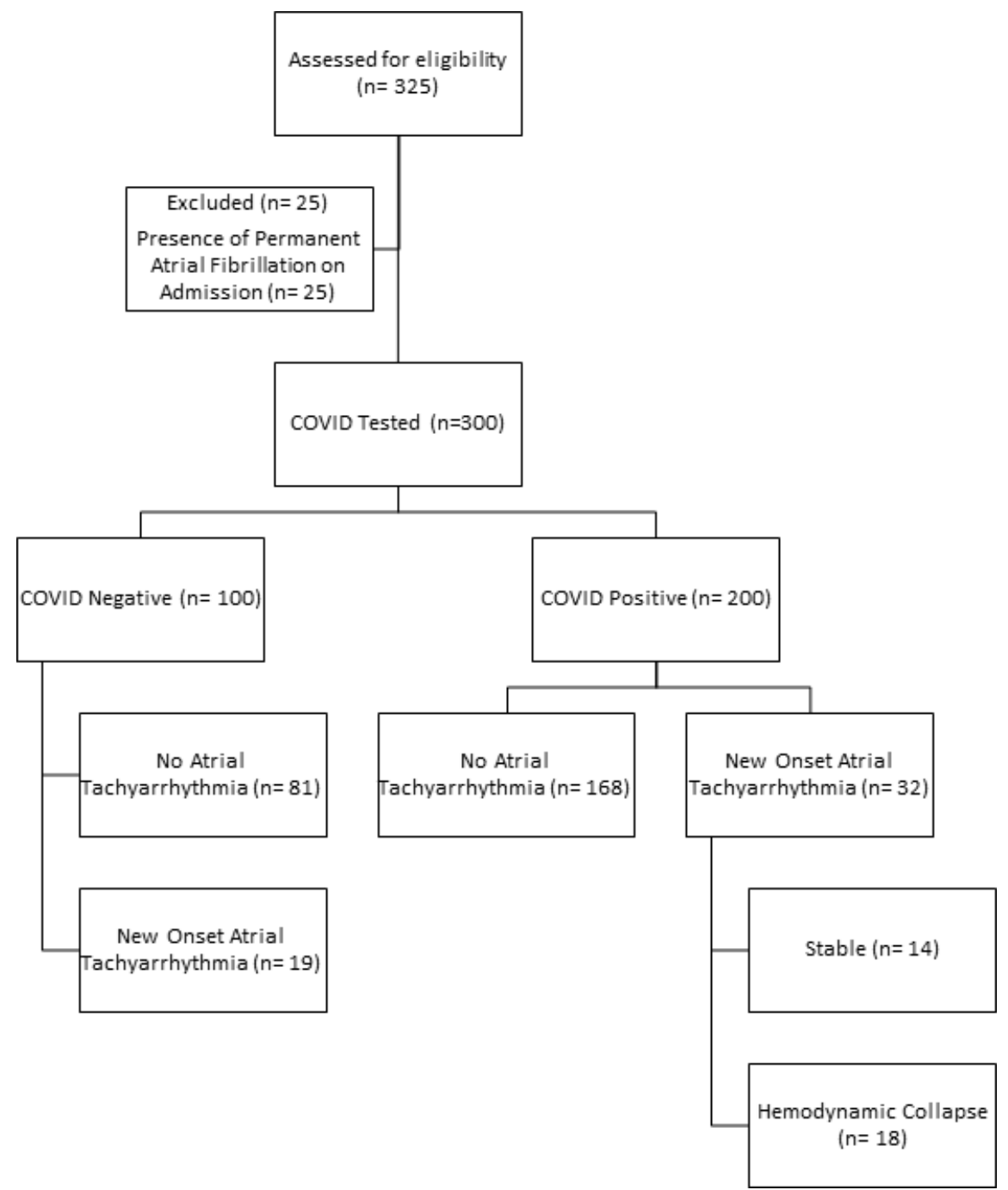

Figure 2:

Norepinephrine Equivalent Doses of Vasopressors in Critically Ill COVID ${ }^{+}$and COVID $^{-}$Individuals in the One Hour Before and the One Hour After Atrial Tachyarrhythmia Onset, February - June 2020 


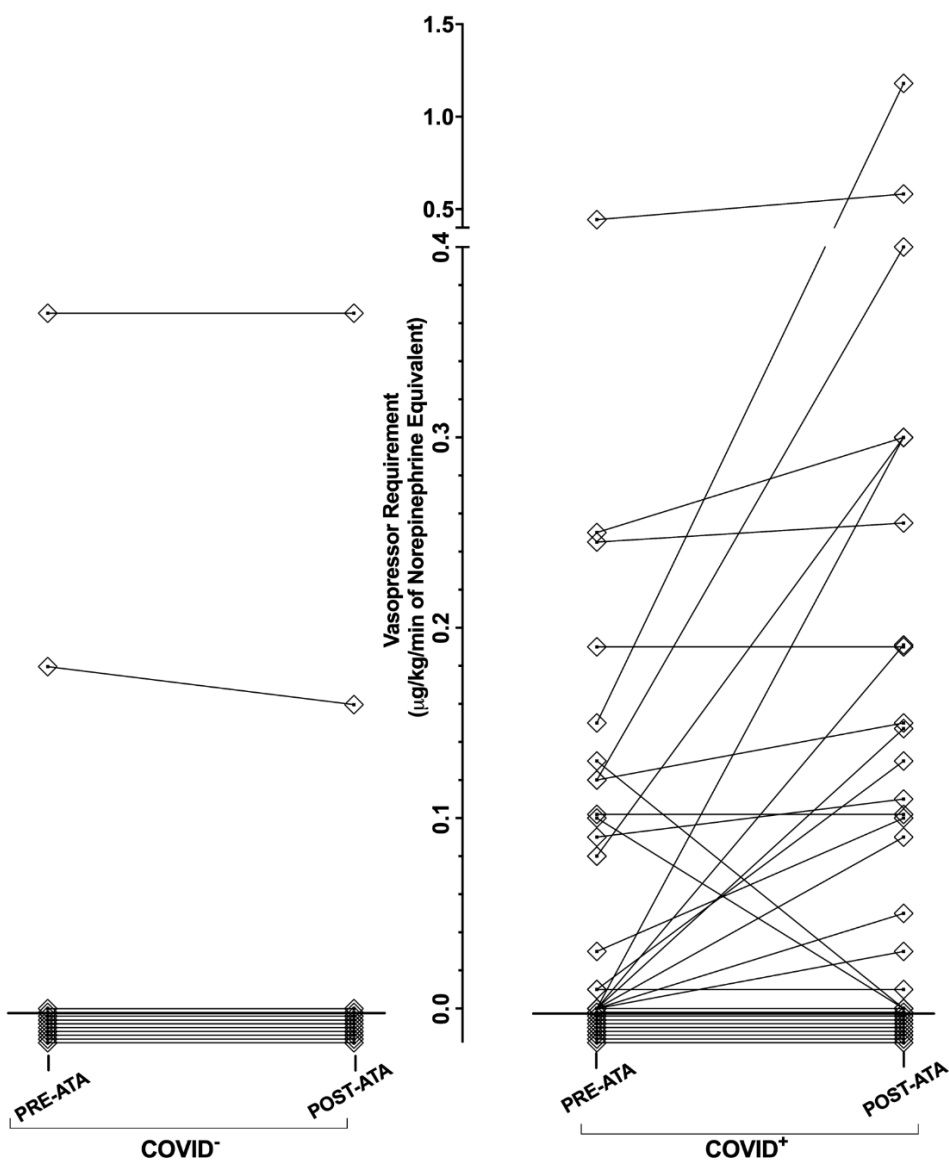

In the COVID- cohort 17 patients had no increase or change in vasopressor use, 1 individual having no change in vasopressor dose, and 1 individual had a decrease in vasopressor dose following ATA onset. In the COVID ${ }^{+}$cohort, 11 individuals were not on a vasopressor and did not require the addition of a vasopressor, 2 individuals had no change in vasopressor dose, 17 individuals saw an increasing vasopressor requirement, 1 individual was taken off vasopressors after ATA onset, and 1 individual was taken off vasopressors after DCCV following ATA onset.

ATA, atrial tachyarrhythmia; COVID, coronavirus disease; DCCV; direct current cardioversion 